\title{
Evaluating the Linearized Almost Ideal Demand System
}

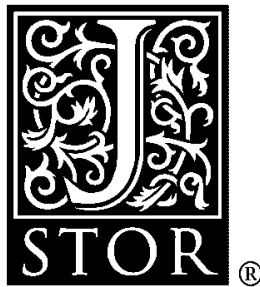

\author{
Adolf Buse
}

American Journal of Agricultural Economics, Vol. 76, No. 4. (Nov., 1994), pp. 781-793.

Stable URL:

http://links.jstor.org/sici?sici=0002-9092\%28199411\%2976\%3A4\%3C781\%3AETLAID\%3E2.0.CO\%3B2-C

American Journal of Agricultural Economics is currently published by American Agricultural Economics Association.

Your use of the JSTOR archive indicates your acceptance of JSTOR's Terms and Conditions of Use, available at

http://www.jstor.org/about/terms.html. JSTOR's Terms and Conditions of Use provides, in part, that unless you have obtained prior permission, you may not download an entire issue of a journal or multiple copies of articles, and you may use content in the JSTOR archive only for your personal, non-commercial use.

Please contact the publisher regarding any further use of this work. Publisher contact information may be obtained at http://www.jstor.org/journals/aaea.html.

Each copy of any part of a JSTOR transmission must contain the same copyright notice that appears on the screen or printed page of such transmission.

The JSTOR Archive is a trusted digital repository providing for long-term preservation and access to leading academic journals and scholarly literature from around the world. The Archive is supported by libraries, scholarly societies, publishers, and foundations. It is an initiative of JSTOR, a not-for-profit organization with a mission to help the scholarly community take advantage of advances in technology. For more information regarding JSTOR, please contact support@jstor.org. 


\title{
Evaluating the Linearized Almost Ideal Demand System
}

\author{
Adolf Buse
}

\begin{abstract}
Linearizing the Almost Ideal Demand System (LAIDS) by recourse to the Stone share weighted price index is common practice. Two issues are addressed here. First, scrutiny of the errors-in-variables implications of the linearization reveals that, not only is the SUR estimator inconsistent, but a consistent IV estimator cannot be constructed. Second, some alternatives to the Green-Alston elasticities specifically designed for the LAIDS model are developed, but neither these nor the Green-Alston elasticities are found to have any advantages over the conventional elasticity formulae. Some errors in the Green-Alston (1990) paper are corrected. The inconsistency and elasticity issues are documented by a Monte Carlo investigation.
\end{abstract}

Key words: Almost Ideal Demand System, inconsistency, instrumental variables, Monte Carlo, seemingly unrelated regressions.

The Almost Ideal Demand System (AIDS) of Deaton and Muellbauer has become the model of choice for many applied demand analysts. It is relatively easy to account for this popularity. The model is grounded in a well-structured analytical framework, accommodates certain types of aggregation, is apparently easy to estimate, and permits testing of the standard restrictions of classical demand theory. Although the model is intrinsically nonlinear, the linearized version of AIDS (LAIDS) using the Stone share weighted price index is widely used to simplify the estimation process. The LAIDS model is the focus of this paper. ${ }^{1}$ I address two principal issues. First, the errors-in-variables implications of the Stone index linearization have never been properly scrutinized. I find that not only is the standard Seemingly Unrelated Regressions (SUR) estimator inconsistent but, within the standard instrumental variable

\footnotetext{
Adolf Buse is professor of economics at the University of Alberta. The author is indebted to Dorothy Golosinski, Ayla Ogus, Yanning Peng, and George Szava-Kovats for research assistance. Helpful advice and useful comments were provided by Julian Alston, John Delehanty, Richard Green, and David Ryan. Alan Sharpe and Noxy Dastoor gave invaluable assistance with programming and computations.

Review coordinated by Steven Buccola.

${ }^{1}$ Between 1980 and 1991 the Deaton-Muellbauer paper was cited 237 times in the Social Sciences Citation Index. Examination of 207 accessible citations revealed that 68 out of 89 empirical applications used the LAIDS specification. In agricultural economics, 23 out of 25 papers chose LAIDS estimation; further details are given in Buse (1993).
}

(IV) framework, a consistent IV estimator cannot be constructed. My second theme is inspired by the recent work of Green and Alston $(1990,1991)$ who have advocated calculation of income and price elasticities specifically designed for the LAIDS model. I extend the Green-Alston approach by developing some alternative LAIDS elasticities, but neither these nor the Green-Alston elasticities are found to have any advantages over conventionally used formulae. As a byproduct of this investigation, I also correct some errors in the 1990 GreenAlston paper. The inconsistency and elasticity issues are addressed by an extensive Monte Carlo investigation.

\section{Estimation}

The AIDS model is defined by

$$
\begin{aligned}
w_{i t}= & \alpha_{i}+\sum_{j}^{n} \gamma_{i j} \ln p_{j t} \\
& +\beta_{i} \ln \left(x_{t} / P_{t}\right)+u_{i t} \quad i=1, \ldots, n \\
\ln P_{t} & =\alpha_{0}+\sum_{j}^{n} \alpha_{j} \ln p_{j t} \\
& +1 / 2 \sum_{i}^{n} \sum_{j}^{n} \gamma_{i j} \ln p_{i t} \ln p_{j t}
\end{aligned}
$$




$$
t=1, \ldots, T
$$

where $w_{i t}$ is the expenditure share of the $i$ th good, $p_{i t}$ is its price, and $x_{t}$ is total expenditure. The adding up conditions are given by

(3a) $\sum_{i}^{n} \alpha_{j}=1$

(3b) $\sum_{i}^{n} \gamma_{i j}=0$

(3c) $\sum_{i}^{n} \beta_{i}=0$

whereas homogeneity and symmetry are defined, respectively, by

$$
\begin{aligned}
& \sum_{j}^{n} \gamma_{i j}=0 \\
& \gamma_{i j}=\gamma_{j i} .
\end{aligned}
$$

The adding-up conditions imply a singular variance-covariance matrix for the disturbances and this is handled in the conventional manner by deleting the $n$th equation. If prices and total expenditure are exogenous, (1) is a nonlinear seemingly unrelated regressions model which can be consistently and asymptotically efficiently estimated by maximum likelihood (ML) with or without the restrictions imposed. Estimating the parameter $\alpha_{0}$ has proved troublesome, so that most of those who employ ML have followed Deaton and Muellbauer and have chosen $\alpha_{0}$ a priori. The resultant conditional ML estimator will be inconsistent to the extent that the chosen $\alpha_{0}$ deviates from its true value, but this is typically ignored in the literature; for further discussion of this point, see Buse (1992).

As noted in the introduction, ML estimation is usually avoided, especially in agricultural economics, in favor of the computationally attractive linearized model. Such a linearization is possible because, apart from $\alpha_{0}$, all model parameters appear in equation (1) so that if $\ln$ $P_{t}$ were known, we would have a set of linear equations which would allow recovery of the entire parametric structure with linear methods. Deaton and Muellbauer exploited this linearity when they suggested that if the prices $\ln p_{j t}$ were highly collinear, then $\ln P_{t}$ could be taken proportional to the observable Stone price index

$$
\ln P_{t}^{*}=\sum_{i}^{n} w_{i t} \ln p_{i t}
$$

There is no reason to suppose that proportionality holds exactly, so we take

$$
P_{t}=\xi_{t} P_{t}^{*}
$$

where $\xi_{t}$ is a random variable with $\mathrm{E}\left(\ln \xi_{t}\right)=$ $\ln \xi_{0}$. If $P_{t}^{*}$ is now used in place of $P_{t}$ in (1), we have the LAIDS model

$$
\begin{aligned}
w_{i t} & =\alpha_{i}^{*}+\sum_{j}^{n} \gamma_{i j} \ln p_{j t} \\
& +\beta_{i} \ln \left(x_{t} / P_{t}^{*}\right)+u_{i t}^{*}
\end{aligned}
$$

where $u_{i t}^{*}=u_{i t}-\beta_{i}\left(\ln \xi_{t}-\ln \xi_{0}\right)$ and $\alpha_{i}^{*}=\alpha_{i}$ $-\beta_{i} \ln \xi_{0}$. This is a classic errors-in-variables problem with $\operatorname{cov}\left(u_{i t}^{*}, \ln P_{t}^{*}\right) \neq 0$. Neither OLS nor SUR, which are often used to estimate (9), is consistent. ${ }^{2}$ The inconsistency has been recognized by Eales and Unnevehr who observe that there is a simultaneity problem insofar as the shares, $w_{i t}$, in the Stone index will be correlated with the disturbances, $u_{i t}$, since $w_{i t}$ is a function of $u_{i t}$. Alston, Foster, and Green have also noted the problem and used 3SLS in their Monte Carlo work to circumvent it.

The textbook solution for errors-in-variables is IV estimation, but that solution does not hold here. In order to make this explicit, first solve for $\ln \xi_{t}=\ln P_{t}-\ln P_{t}^{*}$ by using (1) to construct $\ln P_{t}^{*}=\Sigma w_{i t} \ln p_{i t}$ and then replace $\ln P_{t}$ with (2) to obtain

$$
\begin{aligned}
\ln \xi_{t} & =\alpha_{0}\left(1+\sum_{i} \beta_{i} \ln p_{i t}\right) \\
& +\sum_{i} \beta_{i} \ln p_{i t}\left(\sum_{i} \alpha_{i} \ln p_{i t}-\ln x_{t}\right)
\end{aligned}
$$

\footnotetext{
${ }^{2}$ Although he emphasizes bias, Pashardes comes to the same conclusion by noting that ( 9 ) can be written as an omitted variable problem, namely

$$
w_{i t}=a_{i}+\sum_{j} g_{i j} \ln p_{j t}+\beta_{i} \ln \left(x_{t} / P_{t}^{*}\right)+\beta_{i} \ln \left(P_{t} * / P_{t}\right)+u_{i r}
$$

I am indebted to Chris Nicol for bringing the Pashardes paper to my attention following my presentation of Buse (1992) in Ottawa. Indeed it was the Pashardes paper which prompted me to re-examine my proof of the consistency of the IV estimators I proposed in that paper.
} 


$$
\begin{aligned}
& +1 / 2 \sum_{i} \sum_{j} \gamma_{i j} \ln p_{i t} \ln p_{j t}\left(\sum_{i} \beta_{i} \ln p_{i t}-1\right) \\
& -\sum_{i} u_{i t} \ln p_{i t} .
\end{aligned}
$$

Thus $\ln \xi_{t}=f\left(\ln p_{i t}, \ln x_{t}\right)$ so that the compound disturbance term in (9), $u_{i t}^{*}=u_{i t}-\beta_{i}\left(\ln \xi_{t}\right.$ $\ln \xi_{0}$ ), will be a function of variables which appear as regressors in the equation to be estimated. If we now choose a set of instrumental variables which are correlated with these regressors, they will of necessity be correlated with the disturbances as well. This correlation of instruments and disturbances will violate the standard orthogonality conditions and the IV estimator will be inconsistent. The extent of the inconsistency can in principle be determined analytically, but the algebra is not straightforward because $\ln \xi_{t}$ is not a linear function of $\ln$ $p_{i t}$ and $\ln x_{t}$ but involves cross-product and interaction terms as well. For our purposes, it suffices to note that the IV solution will always fail under standard conditions. We should note that this failure applies regardless of whether we take $\ln p_{i t}$ and $\ln x_{t}$ as exogenous or not. Nor can the problem be finessed by constructing $\ln P_{t}^{*}$ with lagged shares as weights, as has been suggested in a different context by Eales and Unnevehr. With lagged shares as weights, $\ln \xi_{t}$ $=f\left(\ln p_{i t}, \ln p_{i, t-1}, \ln x_{t-1}\right)$ and the presence of $\ln$ $p_{i t}$ in $\ln \xi_{t}$ will again lead to the violation of the orthogonality conditions.

There is one other estimation question to be addressed. The standard AIDS price elasticity formulas require estimates of the $\alpha_{i}$ parameters. In (9) the intercept $\alpha_{i}^{*}=\alpha_{i}-\beta_{i} \ln \xi_{0}$ cannot be used to construct estimators for $\alpha_{i}$ unless we are willing to specify a prior value for $\xi_{0}$. It turns out that the vector $\left(\alpha_{0}, \alpha_{1}, \ldots\right.$, $\alpha_{n}$ ) can readily be estimated from (2) and (3) given that $\gamma_{i j}$ and $\beta_{i}$ have been estimated in (9). Given $\hat{\gamma}_{i j}$ and $\hat{\beta}_{i}$ we can write (2) as

$$
\begin{gathered}
w_{i t}-\hat{a}_{t}-\hat{b}_{t}=-\alpha_{0} \hat{\beta}_{i}+\alpha_{i} \\
-\hat{\beta}_{i} \sum_{j} \alpha_{j} \ln p_{j t}+u_{i t} \\
+\left(a_{t}-\hat{a}_{t}\right)+\left(b_{t}-\hat{b}_{t}\right)
\end{gathered}
$$

where

$$
a_{t}=\sum_{j} \gamma_{i j} \ln p_{j t}
$$

$$
b_{t}=\beta_{i}\left(\ln x_{t}-1 / 2 \sum_{i} \sum_{j} \gamma_{i j} \ln p_{i t} \ln p_{j t}\right)
$$

and $\hat{a}_{t}$ and $\hat{b}_{t}$ are the estimated counterparts. Equation (10) is a linear system in the $\alpha$ 's and can be estimated by SUR or 3SLS. If $\hat{\beta}_{i}$ and $\hat{\gamma}_{i j}$ were consistent, then the last two terms would converge in probability to zero and 3SLS, but not SUR, would be a consistent estimator of the $\alpha$ 's. The SUR estimator would be inconsistent because the $\hat{\beta}_{i}$, which are a component of the regressors, are functions of the $u_{i t}$ and would thus be correlated with the disturbances in (10). Because of the inconsistency of $\hat{\beta}_{i}$ and $\hat{\gamma}_{i j}$ the 3SLS estimator for $\alpha$ will also be inconsistent. ${ }^{3}$

This analysis leads to a simple but uncomfortable conclusion. Not a single parameter estimate reported in the LAIDS literature is based on a consistent estimator. It is, of course, possible for the standard (inconsistent) estimators to have reasonable finite sample properties, and our Monte Carlo experiments will attempt to shed light on this question.

\section{Elasticities}

Uncompensated price elasticities in any demand system are defined by

$$
\eta_{i j}=-\delta_{i j}+\frac{\partial \ln w_{i}}{\partial \ln p_{j}}
$$

where $\delta_{i j}$ is the Kronecker delta and the time subscripts have been suppressed. ${ }^{4}$ It follows directly that if (14) is evaluated using (1), the elasticities for the AIDS model are given by

$$
\begin{aligned}
& \eta_{i j}(A)=-\delta_{i j}+\left(\gamma_{i j} / w_{i}\right) \\
& -\left(\beta_{i} / w_{i}\right)\left(\alpha_{j}+\sum_{k} \gamma_{k j} \ln p_{k}\right) .
\end{aligned}
$$

In the case of LAIDS

\footnotetext{
${ }^{3}$ From the Pashardes paper I discovered that Browning and Meghir proposed an IV estimator that iterated between (8) and (10). They assert that the iterated estimator is consistent but do not provide a proof. Since iteration does not circumvent the problem of misspecification (see footnote 2 ) I conjecture that the iterated estimator will not be consistent

${ }^{4}$ The shares, $w_{i}$, which appear in these equations are, of course, not the observable shares given in (1) but the mean shares $\mathrm{E}\left(w_{i}\right)$. I have suppressed the expectation operator to keep the notation tidy.
} 


$$
\frac{\partial \ln w_{i}}{\partial \ln p_{j}}=w_{i}^{-1}\left(\gamma_{i j}-\beta_{i} \frac{\partial \ln P^{*}}{\partial \ln p_{j}}\right)
$$

and we must make a choice as to how to evaluate $\partial \ln P * / \partial \ln p_{j}$. The simplest procedure is to argue that since $P^{*}$ is presumed to be a good approximation to $P$, then $\partial \ln P * / \partial \ln p_{j}$ will be well approximated by $\partial \ln P / \partial \ln p_{j}$. Thus, we would use (15) to calculate elasticities for LAIDS. However, from (7) we have that

$$
\frac{\partial \ln P^{*}}{\partial \ln p_{j}}=w_{j}+\sum_{k} w_{k} \ln p_{k} \frac{\partial \ln w_{k}}{\partial \ln p_{j}}
$$

and this leads to two different definitions of LAIDS elasticities depending on whether (1) or (9) is used to evaluate $\left(\partial \ln w_{k} / \partial \ln p_{j}\right)$ in (17).

Holding to the LAIDS framework would mean choosing (9) to give

$$
\begin{aligned}
& \frac{\partial \ln P^{*}}{\partial \ln p_{j}} \\
& =\left(w_{j}+\sum_{k} \gamma_{k j} \ln p_{k}\right)\left(1+\sum_{k} \beta_{k} \ln p_{k}\right)^{-1} .
\end{aligned}
$$

The elasticities are then given by

$$
\begin{aligned}
\eta_{i j}\left(L_{1}\right) & =-\delta_{i j}+\left(\gamma_{i j} / w_{i}\right) \\
& -\left(\beta_{i} / w_{i}\right)\left(w_{j}+\sum_{k} \gamma_{k j} \ln p_{k}\right) \\
& \left(1+\sum_{k} \beta_{k} \ln p_{k}\right)^{-1} .
\end{aligned}
$$

It turns out, perhaps not surprisingly, the $\eta_{i j}\left(L_{1}\right)$ elasticities are the LAIDS elasticities given by Green and Alston (1990, p. 443) as

$$
\begin{aligned}
& \eta_{i j}\left(L_{1}\right)=-\delta_{i j}+\left(\gamma_{i j} / w_{i}\right) \\
& \quad-\left(\beta_{i} / w_{i}\right)\left[w_{j}+\sum_{k} w_{k} \ln p_{k}\left(\eta_{k j}+\delta_{k j}\right)\right] .
\end{aligned}
$$

In their formulation, $\eta_{i j}$ appears on the righthand side of (19a) and the solution for $\eta_{i j}$ comes from a system of simultaneous equations which solves for all the elasticities jointly. The equivalence of the two definitions can be established by using (19) to define $\eta_{k j}+\delta_{k j}$, substituting in (19a) and observing that (19a) then reproduces (19); that is, the two definitions are internally consistent.

If $\partial \ln P * / \partial \ln p_{j}$ is viewed as an approximation to $\partial \ln P / \partial \ln p_{j}$, then using (1), which is the model we are trying to approximate, may prove to be a better numerical procedure for evaluating $\left(\partial \ln w_{k} / \partial \ln p_{j}\right)$ in (17). This choice gives

$$
\begin{aligned}
& \frac{\partial \ln P^{*}}{\partial \ln p_{j}}=w_{j}+\sum_{k} \gamma_{k j} \ln p_{k} \\
& -\sum_{k} \beta_{k} \ln p_{k}\left(\alpha_{j}+\sum_{k} \gamma_{k j} \ln p_{k}\right)
\end{aligned}
$$

and the corresponding elasticities as

$$
\begin{aligned}
\eta_{i j}\left(L_{2}\right) & =-\delta_{i j}+\left(\gamma_{i j} / w_{i}\right) \\
& -\left(\beta_{i} / w_{i}\right)\left[w_{j}+\sum_{k} \gamma_{k j} \ln p_{k}\right. \\
& \left.-\sum_{k} \beta_{k} \ln p_{k}\left(\alpha_{j}+\sum_{k} \gamma_{k j} \ln p_{k}\right)\right]
\end{aligned}
$$

We can note that if $\sum_{k} \beta_{k} \ln p=0$, then $\eta_{i j}\left(L_{1}\right)$ $=\eta_{i j}\left(L_{2}\right)$ and this will hold for at least one sample point if prices are normalized at $p_{k}=1$ $(k=1, \ldots, n)$ for one time period. Furthermore, when $p_{k}=1, \sum_{k} \gamma_{k j} \ln p_{k}=0$, and $\eta_{i j}\left(L_{1}\right)=$ $\eta_{i j}\left(L_{2}\right)$ are also equal to

$$
\eta_{i j}\left(L_{3}\right)=-\delta_{i j}+\left(\gamma_{i j} / w_{i}\right)-\beta_{i}\left(w_{j} / w_{i}\right)
$$

which is an alternative definition for LAIDS elasticities found in the literature (Goddard) and numerically evaluated by Green and Alston. This variant can also be obtained from (17) by taking $\partial \ln P * / \partial \ln P=w_{j}$, and can be considered the most intuitively natural definition. ${ }^{5}$

There is one other possibility. At unit prices, $\ln P=\alpha_{0}$ and $\ln P^{*}=0$, so that at the mean in (8) $\ln \xi_{0}=\alpha_{0}$, where $\alpha_{0}$ is interpreted as the logarithm of subsistence expenditure at unit prices; see Deaton and Muellbauer (p. 316). Furthermore, at unit prices (1) can be written as $w_{i}=\alpha_{i}+\beta_{i} \ln x-\beta_{i} \alpha_{0}$ so that if we choose $\xi_{0}$ as the expenditure level in the base period, then ln $x=\ln \xi=\alpha_{0}$ and $w_{i}=\alpha_{i}$. An approximation to

\footnotetext{
${ }^{5}$ Chalfant is most often cited in this context although, as best as I can determine, Goddard was the first to derive $\eta_{i j}\left(L_{3}\right)$. Another variant of the elasticities is obtained by taking $\beta_{i}=0$ (homotheticity) so that $\eta_{i j}\left(L_{4}\right)=-\delta_{i j}+\left(\gamma_{i j} / w_{i}\right)$, but this is likely to be of limited relevance as there is little empirical justification for imposing homotheticity.
} 
(15) can then be constructed by replacing $\alpha_{j}$ with $w_{j}$ to give the elasticity

$$
\begin{aligned}
\eta_{i j}\left(A_{0}\right) & =-\delta_{i j}+\left(\gamma_{i j} / w_{i}\right) \\
& -\left(\beta_{i} / w_{i}\right)\left(w_{j}+\sum_{k} \gamma_{k j} \ln p_{j}\right) .
\end{aligned}
$$

Alternatively, $\eta_{i j}\left(A_{0}\right)$ can also be viewed as a truncated version of $\eta_{i j}\left(L_{1}\right)$ and $\eta_{i j}\left(L_{2}\right)$ in which all expressions in the last term of (18) and (21) other than $\left(\beta_{i} / w_{i}\right)\left(w_{j}+\sum_{k} \gamma_{k j} \ln p_{j}\right)$ have been omitted. Inasmuch as $\eta_{i j}\left(A_{0}\right)$ is predicated on unit prices, it would not be appropriate to use $\eta_{i j}\left(A_{0}\right)$ when prices are not normalized, as is the case in some empirical studies. ${ }^{6}$

Whether or not one chooses to treat any one of these elasticities as correct is a matter of perspective. If the LAIDS model is viewed as the data generating process, it is indeed the case that $\eta_{i j}\left(L_{1}\right)$ would give the correct elasticities. But LAIDS is not an integrable demand system and is almost invariably viewed as an approximation procedure designed to circumvent problems of convergence in estimating the AIDS model. Indeed, we have not found a single case in the literature in which the LAIDS specification was taken as the analytical model for the consumer's choice problem. By definition any approximation procedure will induce errors, apart from the inconsistency already noted, and we cannot a priori define any one procedure as correct. What we must do is choose among the alternatives on the basis of their ability to approximate the true values of the parameters of interest. Their characterization of $\eta_{i j}\left(L_{1}\right)$ as the correct elasticity notwithstanding, Green and Alston take this position when they evaluate how close the different elasticity formulas come to those obtained from (conditional) maximum likelihood estimation of the AIDS model using the Blanciforti, Green, and King data.

My survey of the empirical literature has allowed me to cull some evidence on common practice with respect to elasticity calculations. Many studies fail to specify the elasticity formula, but from those which did I obtained the following frequency of use of price elasticities (in decreasing order of frequency): (a)

\footnotetext{
${ }^{6}$ The elasticity definitions given in Murfin also allow one to write $(15)$ as $\eta_{i j}(A)=-\delta_{i j}+\left(\gamma_{i j} / w_{i}\right)-\left(\beta_{i} / w_{i}\right)\left[w_{j}-\beta_{j} \ln (x / P)\right]$. By substituting the Stone index $P *$ for $P$, we have another operational AIDS elasticity estimator which finesses the problem of estimating the $\alpha_{i}$. Unfortunately, such a possibility came to my attention after the Monte Carlo work was completed.
}

$\eta_{i j}\left(L_{3}\right)-26,(b) \eta_{i j}(A)-19,(c) \eta_{i j}\left(L_{4}\right)-4$, and $(d) \eta_{i j}\left(L_{1}\right)-2$. These numbers are, presumably, no surprise to those active in this area, but it is worth noting that the Green and Alston elasticities have made an appearance in the literature.

Green and Alston (1991) argue that the conventional AIDS income elasticity

$$
\eta_{i x}(A)=1+\left(\beta_{i} / w_{i}\right)
$$

is also invalid in the LAIDS model. Arguments (Buse 1993) analogous to those made in deriving the price elasticities give two alternative income elasticities

$$
\begin{aligned}
\eta_{i x}\left(L_{1}\right) & =1 \\
& +\left(\beta_{i} / w_{i}\right)\left[1-\frac{\sum_{k} \beta_{k} \ln p_{k}}{1+\sum_{k} \beta_{k} \ln p_{k}}\right]
\end{aligned}
$$

$$
\begin{aligned}
\eta_{i x}\left(L_{2}\right) & =1 \\
& +\left(\beta_{i} / w_{i}\right)\left[1-\sum_{k} \beta_{k} \ln p_{k}\right]
\end{aligned}
$$

The first is equivalent to the income elasticity proposed by Green and Alston. Again there is no basis for a prior claim that either (25) or (26) is the correct LAIDS income elasticity. The question remains empirical rather than analytical.

The relative merits of the elasticity formulas will be explored in depth in my Monte Carlo experiments. Before I do so, I need to digress briefly to examine the illustrative calculations of uncompensated own-price elasticities presented by Green and Alston (1990). I have attempted to replicate their results but have not been entirely successful, although the numerical differences in our respective estimates were small, differing typically only in the third decimal place. In the course of this replication exercise I discovered three features of the Green and Alston computations that require correction. First, the ML/AIDS elasticities recorded in the first row of their table 1 are based on thirty-one observations for the period 1948-78, whereas the LAIDS elasticities were calculated for 1947-78 (thirty-two observations). Second, Green and Alston did not follow conventional practice and normalize all the price indices at unity, but used the original series with a base of 100 in 1972. Third, in computing LAIDS elas- 
Table 1. Uncompensated Price Elasticities 1947-78 Under Homogeneity and Symmetry

\begin{tabular}{l|lrrrr}
\hline & $\eta_{i i}(\cdot)^{\mathrm{a}}$ & $\eta_{11}^{\mathrm{b}}$ & $\eta_{22}$ & $\eta_{33}$ & $\eta_{44}$ \\
\hline AIDS (ML) & & & & & \\
1. 47-48 & $\eta_{i i}(\mathrm{~A})$ & -1.011 & -0.244 & -0.807 & -0.789 \\
2. Green-Alston (48-78) & $\eta_{i i}(\mathrm{~A})$ & -0.944 & -0.256 & -0.799 & -0.787 \\
\hline LAIDS (SUR) & & & & & \\
3. In $p_{j}=1.0(1972)$ & $\eta_{i i}\left(\mathrm{~A}^{*}\right)$ & 1.146 & -0.159 & -0.532 & 0.859 \\
4. Green-Alston (1972=100) & $\eta_{i i}\left(\mathrm{~A}^{*}\right)$ & -0.411 & -0.229 & -0.736 & -0.325 \\
5. & $\eta_{i i}(\mathrm{~A})$ & -1.009 & -0.256 & -0.814 & -0.780 \\
6. Green-Alston & $\eta_{i i}\left(\mathrm{~L}_{1}\right)$ & -0.996 & -0.255 & $-0.791^{\mathrm{c}}$ & -0.761 \\
7. & $\eta_{i i}\left(\mathrm{~L}_{3}\right)$ & -0.988 & -0.255 & -0.811 & -0.764 \\
8. & $\eta_{i i}\left(\mathrm{~L}_{4}\right)$ & -0.644 & -0.200 & -0.888 & -1.066 \\
\hline
\end{tabular}

a All elasticities evaluated at sample means.

b The four expenditure groups are: (1) Meats, (2) Fruits and Vegetables, (3) Cereal and Bakery Products, (4) Miscellaneous Foods.

c This figure replaces the incorrect value of -0.810 given in Green and Alston (1990, table 1, p. 444).

ticities using the AIDS definition given by equation (15), Green and Alston used $\hat{\alpha}_{i}^{*}$ as the estimate of $\alpha_{i}$ instead of the correct estimate given by $\hat{\alpha}_{i}=\hat{\alpha}_{i}^{*}+\hat{\beta}_{i} \ln \xi_{0}$; see equation (9) above.

The implications of these points for the Green and Alston elasticities are recorded in table 1. The first row gives the AIDS ML elasticities for the complete period 1947-78 with Green and Alston's 1948-78 elasticities immediately below for comparison. The differences are modest, but the full-sample estimates do provide the correct benchmark for the LAIDS elasticities reported by them. More important, both sets of AIDS ML elasticities were derived from estimates made with prices normalized at unity in 1972; the reported Green and Alston ML elasticities are those given in Blanciforti, Green, and King (p. 36). The LAIDS model should therefore be estimated under this normalization, as the estimated intercepts, $\hat{\alpha}_{i}^{*}$ in (9), depend on the choice of normalization. Reestimation is not required since the intercepts under unit normalization can be recovered by subtracting $\hat{\beta}_{i} \ln 100$ from Green and Alston's $\hat{\alpha}_{i}^{*}$. When this is done, the LAIDS elasticities denoted by $\eta_{i j}\left(A^{*}\right)$ change dramatically; compare the third and fourth row of table 1. Indeed, with two positive own-price elasticities, the corrected elasticities deviate from the benchmark ML elasticities by even greater amounts than recorded in Green and Alston. This appears to add even greater force to Green and Alston's warning about the deficiencies of using the AIDS formula (15) in the LAIDS context.

That warning is, however, misplaced. In making the calculations above, I have followed
Green and Alston and substituted $\hat{\alpha}_{i}^{*}$ into (15) rather than use an estimate of $\alpha_{i}$ as specified in the definition of the $\eta_{i j}(A)$ elasticity. Since $\alpha_{i}^{*}=\alpha_{i}-\beta_{i} \ln \xi_{0}$, it is immediately apparent that using $\alpha_{i}^{*}$ will generate systematically biased estimates of the elasticities (over and above the inconsistency problem discussed earlier). The direction of the bias is readily determined on noting that $\eta_{i j}\left(A^{*}\right)=\eta_{i j}(A)+\left(\beta_{i} \beta_{j}\right.$ ln $\xi_{0} / w_{i}$ ) so that on taking $\ln \xi=\alpha_{0}>0$ we have $\eta_{i i}\left(A^{*}\right)=\eta_{i i}(A)$ for $i=j$. This accounts for the observed positive own-price elasticities discussed in the previous paragraph. ${ }^{7}$ We can estimate $\hat{\alpha}_{i}=\hat{\alpha}_{i}^{*}+\hat{\beta}_{i} \ln \xi_{0}$ once the subsistence level is chosen. In Blanciforti, Green, and King (p. 40) it was chosen as ln $\$ 586.90$, the log of per capita expenditure in the reference year 1972. Green and Alston's ML estimates, as well as mine, were conditioned on this value. We therefore estimated $\alpha_{i}$ by $\hat{\alpha}_{i}=\hat{\alpha}_{i}^{*}+\hat{\beta}_{i} \ln$ (5.869) and then recalculated the elasticities. The results in row five of table 1 show that these elasticities give the best approximations to the benchmark ML elasticities recorded in the first row. The Green-Alston critique of the elasticity is therefore unfounded, resting as it does on an inappropriate choice of a parameter estimate.

With the exception of the first two rows, all of the elasticities reported in table 1 were derived from Green and Alston's SHAZAM esti-

\footnotetext{
${ }^{7}$ Fulponi, Haden, and Mergos; and Donatos all give $\eta_{i j}(A)$ as the elasticity formula but estimate the LAIDS model. None of them indicate that any adjustments were made to the estimated intercepts in order to obtain the $\alpha_{i}$ needed for $\eta_{i j}(A)$. We have to assume that the incorrect $\alpha_{i}^{*}$ were used, generating a corresponding error in the reported elasticities.
} 
mates, which I was unable to replicate. I have re-estimated the model for the period 1948-78, using both SUR and 3SLS, and calculated all elasticities including $\eta_{i j}\left(A_{0}\right)$ and $\eta_{i j}\left(L_{2}\right)$ newly developed here. Relative to the ML estimates, the results indicate that an investigator would not go seriously astray using $\eta_{i i}(A)$ or any one of $\eta_{i i}\left(L_{j}\right), j=1,2,3$. However, the best results are obtained with $\eta_{i j}(A)$, whereas $\eta_{i j}\left(A^{*}\right)$, using the incorrect intercept estimates, is simply disastrous.

\section{Monte Carlo Design}

Space limitations preclude a detailed account of the Monte Carlo design and the results. What follows in the next two sections is, therefore, an overview and summary, with full detail available in Buse (1993). A number of considerations, both theoretical and empirical, played a role in the Monte Carlo design. At the theoretical level, I wanted to choose a structure that satisfied the regularity conditions for a demand system. At the empirical level, I wanted to use data generating processes which captured some of the characteristics of actual data sets and which generated elasticities comparable in magnitude to those that might be observed in a typical demand study. These objectives were pursued using a four-equation system of demand equations, as this is the system size most often estimated.

Imposing adding up, homogeneity, and symmetry is relatively straightforward and in everything which follows, including the estimation in the next section, these restrictions have been imposed. In the AIDS model, negativity (negative semi-definiteness of the Slutsky substitution matrix) cannot be imposed globally, so I imposed it at a particular reference point, namely at unit prices. The analysis starts with the elasticity form of the Slutsky equation

$$
\eta_{i j}=\left(p_{j} / q_{i}\right) s_{i j}-w_{j} \eta_{i x}
$$

where $s_{i j}$ is the matrix of substitution terms. Following Deaton and Muellbauer's (p. 316) suggestion, we work with $s_{i j}^{*}=p_{i} p_{j} s_{i j} / x$, so that (27) becomes

$$
\eta_{i j}=\left(s_{i j}^{*} / w_{i}\right)-w_{j} \eta_{i x}
$$

where $s_{i j}^{*}$ is defined by

$$
\begin{aligned}
s_{i j}^{*} & =\gamma_{i j} \\
& +\beta_{i} \beta_{j} \ln (x / P)+w_{i} \delta_{i j}+w_{i} w_{j} .
\end{aligned}
$$

The AIDS cost function is given by

$$
\begin{aligned}
\ln c(U, p) & \equiv \ln x \\
& =\ln P+U \beta_{0} \exp \left(\sum_{k} \beta_{k} \ln p_{k}\right)
\end{aligned}
$$

so that on choosing $U=1$ and $\beta_{0}=1$ we have, at unit prices, that $\ln (x / P)=1$. Specifying $\beta_{i}$, $w_{i}$, and $\gamma_{i j}$ would then allow us to determine $s_{i j}^{*}$ and $\eta_{i j}$. I did not proceed in this fashion, however, but specified $s_{i j}^{*}$ first in order to ensure negative semidefiniteness, and then determined $\gamma_{i j}$ residually, given the chosen vectors $\boldsymbol{\beta}=(0.2$, $0.1,-0.1,-0.2)$ and $\mathbf{w}=(0.35,0.2,0.2,0.25)$.

Two substitution matrices were specified, one with a pair of complementary goods and the other with a pair of independent goods. The absolute value of the substitution effects were generally larger in the "complementary goods" matrix. The vector of income elasticities was set at $\eta_{x}=(1.57,1.5,0.5,0.2)$ and (28) was then used to determine two matrices of uncompensated price elasticities. In the independent goods case the own-price elasticities varied between -0.290 and -0.836 and the cross-price elasticities ranged from -0.393 to 0.160 . For the complementary case own-price elasticities varied from -0.490 to -1.100 and cross-price elasticities between -0.450 and 0.320 . The parametric structure was completed by taking $\alpha_{0}=$ 1 and using the share equation at unit prices, $w_{i}$ $=\alpha_{i}+\beta_{i}$, to determine $\alpha=(0.15,0.1,0.3$, $0.45)$.

Log prices were generated by $\mathrm{AR}(1)$ processes with means and variances comparable to those found in the literature. The degree of collinearity among prices was determined by the covariances of the white noise across the AR(1) processes. Two polar cases of collinearity were specified: no collinearity and high collinearity. The correlation structure for log prices in the high collinearity case was as follows: $r_{12}=$ $0.99, r_{13}=r_{23}=0.98, r_{14}=r_{24}=r_{34}=0.97$. Correlations of this order of magnitude are common, and such a specification will provide a check on Deaton and Muellbauer's claim that high collinearity between prices will make the Stone price index a good approximation to $\ln P_{t}$.

To complete the data set, the process generating the total expenditure variable must be 
specified. Given that $\ln x_{t}=\ln P_{t}+U \beta_{0} \exp$ $\left(\Sigma \beta_{k} \ln p_{k t}\right), \ln \left(x_{t} / P_{t}\right)$ was generated by setting $\beta_{0}=1$ and letting

$$
U_{t}=1.0+v_{t}
$$

with $v_{t} \sim N(0,0.0001)$. This specification introduces variation in utility that might realistically be expected in a time series context.

I generated 201 observations on prices and utility. The first 80 observations were deleted to make the prices in the autoregressive structure independent of starting conditions. The remaining 121 observations were used to construct sample sizes of 30,60 , and 120 , the extra observation being used up in the creation of lagged instruments. Observed shares were then generated by adding the disturbances to the systematic component. The disturbances vector was taken as $\mathbf{u} \sim N(0, \boldsymbol{\Sigma})$. Two variance-covariance structures were specified to determine what effect increasing disturbance variance had on the properties of the estimators and elasticities. Recall from (10) that the variance of the measurement error in $\ln P_{t}^{*}$ is directly proportional to the variance of $u_{i t}$, so that by increasing the variances, the size of the inconsistency of the estimator should increase. The first set of variances was chosen to generate goodness-offit statistics in the individual equations corresponding to those commonly observed in the literature. Thus, with one exception, at least two of the three $R^{2}$ 's in each experiment were greater than 0.70 and overall more than half of them were greater than 0.90 . The $\mathrm{R}^{2}$ statistics were generated with cross equation correlations of $r_{12}=0.5, r_{13}=0.6$, and $r_{23}=0.7$. These correlations were maintained in the high variance experiment and under these conditions all the $\mathrm{R}^{2}$ 's, with two exceptions, were less than 0.4 .

With two substitution matrices, two price specifications (correlated and uncorrelated), three sample sizes $(30,60,120)$, and two disturbance variance-covariance matrices (small and large variances), there were a total of twenty-four experiments. Each of these structures were also estimated by SUR and 3SLS so that a total of forty-eight experiments were executed. The number of replications was taken to be one thousand. All estimation and computation was done with TSP 4.2.

It is necessary to define the criteria which will be used to evaluate the Monte Carlo output. The system has a very large number of parameters and it would not be instructive to present the conventional bias and mean square error for each coefficient or elasticity. Some data reduction is essential to allow the reader to grasp the broad patterns. I have chosen two summary measures, aggregate bias and trace mean square error. Let $\boldsymbol{\theta}$ be a vector of true parameter values and $\hat{\boldsymbol{\theta}}$ the mean of the distribution of the estimated parameters. If $\boldsymbol{B}(\hat{\boldsymbol{\theta}})=\hat{\boldsymbol{\theta}}-$ $\boldsymbol{\theta}$ is the bias, then the aggregate bias is defined by

$$
\begin{aligned}
A B & =(\hat{\boldsymbol{\theta}}-\boldsymbol{\theta})^{\prime}(\hat{\boldsymbol{\theta}}-\boldsymbol{\theta}) \\
& =B(\hat{\boldsymbol{\theta}})^{\prime} B(\hat{\boldsymbol{\theta}})
\end{aligned}
$$

which is the sum of the squared biases. The MSE of the vector is defined by MSE $=\operatorname{var}(\hat{\boldsymbol{\theta}})$ $+B(\hat{\boldsymbol{\theta}}) B(\hat{\boldsymbol{\theta}})^{\prime}$, so that the trace MSE $(T M)$ is given by

$$
T M=\operatorname{trvar}(\hat{\boldsymbol{\theta}})+A B
$$

Thus, the trace MSE will give the usual combination of variance and bias in assessing performance. These criteria will be applied to the parameter vector $\boldsymbol{\theta}=(\boldsymbol{\alpha}, \boldsymbol{\beta}, \boldsymbol{\gamma})$ of the AIDS model, as well as to the various elasticities which are functions of these parameters.

A final point needs to be made about the true elasticities which were used in the evaluations. Because the elasticities are to be evaluated at the sample means, we need corresponding population mean values. In particular we need

$$
\mathrm{E}\left(w_{i}\right)-\alpha_{i}+\sum_{j} \gamma_{i j} \mathrm{E}\left(\ln p_{j}\right)+\beta_{i} \mathrm{E}[\ln (x / P)] .
$$

Means of the $\log$ prices are given by the autoregressive structure and $\mathrm{E}[\ln (x / P)]=$ $\mathrm{E}(U) \mathrm{E}\left[\exp \left(\sum \beta_{k} \ln p_{k}\right)\right]$, where the stochastic component of $U$ and log prices are known to be independent. $\mathrm{E}(U)=1$ from (28), but in order to evaluate $\mathrm{E}\left[\exp \left(\sum \beta_{k} \ln p_{k}\right)\right]=\mathrm{E}\left(\Pi p_{k}^{\beta_{k}}\right)$ we must invoke Theorem 2.4 (Aitchison and Brown, p. 12) on the distribution of products of lognormal variables. The analytical convenience of log-normality should be apparent. Analytical evaluation of expressions such as $\mathrm{E}\left(\ln p_{j}\right)$ and $\mathrm{E}\left(\Pi p_{k}^{\beta_{k}}\right)$ under normality of prices is not feasible. Of course, log-normality also guarantees positive prices.

\section{Results}

Although I indicated earlier that a detailed presentation of sampling means and variances for individual coefficients would be impractical, I 
Table 2. Means and Standard Deviations of Sampling Distribution of Parameters and Aggregate Bias and Trace Mean Square Error

\begin{tabular}{|c|c|c|c|c|c|c|c|}
\hline & \multirow[b]{2}{*}{ True value } & \multicolumn{3}{|c|}{ SUR $^{d}$} & \multicolumn{3}{|c|}{$3 S_{L S}^{d}$} \\
\hline & & $T=30$ & $T=60$ & $T=120$ & $T=30$ & $T=60$ & $T=120$ \\
\hline \multirow[t]{2}{*}{$\alpha_{0}$} & 1.0000 & 1.9986 & 2.0013 & 2.0070 & 2.0160 & 2.0067 & 2.0119 \\
\hline & & 0.0029 & 0.0011 & 0.0008 & 0.0412 & 0.0328 & 0.0250 \\
\hline \multirow[t]{2}{*}{$\alpha_{1}$} & 0.1500 & 0.3502 & 0.3505 & 0.3514 & 0.3519 & 0.3503 & 0.3513 \\
\hline & & 0.0016 & 0.0005 & 0.0000 & 0.0065 & 0.0031 & 0.0028 \\
\hline \multirow[t]{2}{*}{$\alpha_{2}$} & 0.1000 & 0.1982 & 0.1983 & 0.2014 & 0.1998 & 0.2002 & 0.2007 \\
\hline & & 0.0006 & 0.0002 & 0.0002 & 0.0052 & 0.0039 & 0.0024 \\
\hline \multirow[t]{2}{*}{$\alpha_{3}$} & 0.3000 & 0.2008 & 0.2000 & 0.1994 & 0.1981 & 0.1993 & 0.1989 \\
\hline & & 0.0005 & 0.0000 & 0.0003 & 0.0048 & 0.0031 & 0.0031 \\
\hline \multirow[t]{2}{*}{$\beta_{1}$} & 0.2000 & 0.2247 & 0.2450 & 0.2002 & 0.1998 & 0.2464 & 0.1874 \\
\hline & & 0.0104 & 0.0022 & 0.0008 & 0.1158 & 0.2168 & 0.1215 \\
\hline \multirow[t]{2}{*}{$\beta_{2}$} & 0.1000 & 0.1263 & 0.1612 & 0.1230 & 0.1137 & 0.1507 & 0.1052 \\
\hline & & 0.0118 & 0.0328 & 0.0116 & 0.1645 & 0.3402 & 0.1862 \\
\hline \multirow[t]{2}{*}{$\beta_{3}$} & -0.1000 & -0.0815 & -0.0537 & -0.0876 & -0.0945 & -0.0588 & -0.0964 \\
\hline & & 0.0441 & 0.0018 & 0.0192 & 0.0873 & 0.1660 & 0.0959 \\
\hline \multirow[t]{2}{*}{$\gamma_{11}$} & 0.0875 & 0.1307 & 0.1298 & 0.1296 & 0.1268 & 0.1266 & 0.1271 \\
\hline & & 0.0034 & 0.0031 & 0.0011 & 0.0106 & 0.0046 & 0.0030 \\
\hline \multirow[t]{2}{*}{$\gamma_{12}$} & -0.0300 & -0.0173 & -0.0140 & -0.0075 & -0.0090 & -0.0090 & -0.0092 \\
\hline & & 0.0042 & 0.0005 & 0.0003 & 0.0104 & 0.0053 & 0.0028 \\
\hline \multirow[t]{2}{*}{$\gamma_{13}$} & -0.0100 & -0.0301 & -0.0302 & -0.0295 & -0.0304 & -0.0299 & -0.0300 \\
\hline & & 0.0007 & 0.0004 & 0.0003 & 0.0053 & 0.0026 & 0.0017 \\
\hline \multirow[t]{2}{*}{$\gamma_{22}$} & 0.0700 & 0.0793 & 0.0837 & 0.0780 & 0.0801 & 0.0799 & 0.0806 \\
\hline & & 0.0071 & 0.0035 & 0.0021 & 0.0123 & 0.0081 & 0.0037 \\
\hline \multirow[t]{2}{*}{$\gamma_{23}$} & -0.0200 & -0.0335 & -0.0341 & -0.0301 & -0.0311 & -0.0317 & -0.0317 \\
\hline & & 0.0008 & 0.0019 & 0.0016 & 0.0061 & 0.0036 & 0.0021 \\
\hline \multirow[t]{2}{*}{$\gamma_{33}$} & 0.0500 & 0.0617 & 0.0593 & 0.0593 & 0.0618 & 0.0615 & 0.0620 \\
\hline & & 0.0026 & 0.0004 & 0.0005 & 0.0044 & 0.0027 & 0.0019 \\
\hline $\mathbf{R}_{*}^{2 \mathrm{a}}$ & & 0.9955 & 0.9966 & 0.9948 & 0.9955 & 0.9966 & 0.9948 \\
\hline $\mathrm{AB} 1^{\mathrm{b}}$ & & 0.0045 & 0.0108 & 0.0036 & 0.0030 & 0.0092 & 0.0030 \\
\hline $\mathrm{AB} 2^{\mathrm{c}}$ & & 1.0567 & 1.0625 & 1.0750 & 1.0933 & 1.0738 & 1.0848 \\
\hline $\mathrm{TM}^{\mathrm{b}}$ & & 0.0068 & 0.0124 & 0.0041 & 0.1515 & 0.1996 & 0.0617 \\
\hline $\mathrm{TM}^{\mathrm{c}}$ & & 1.0567 & 1.0625 & 1.0750 & 1.0951 & 1.0749 & 1.0855 \\
\hline
\end{tabular}

${ }^{a}$ Average correlation (squared) between $P_{t}$ and $P_{t}^{*}$ across 1,000 replications.

${ }^{b}$ Aggregate bias and trace MSE for $\beta$ and $\gamma$ coefficients.

${ }^{c}$ Aggregate bias and trace MSE for $\alpha$ coefficients.

${ }^{d}$ All elasticities evaluated at sample means.

begin the presentation of the results by examining in detail one experiment. This will serve as an illustration to give some feel for the order of magnitude of the numbers. Table 2 gives the means and standard deviations of the sampling distribution of the SUR and 3SLS estimators for the independent goods case with highly correlated prices and good equation fit. Under these conditions $\ln P_{t}^{*}$ is a good approximation to $\ln P_{t}$ and the squared correlation between the two variables, $R_{*}^{2}$, is greater than 0.99 at all sample sizes. Because the $\beta_{i}$ and $\gamma_{i j}$ are estimated first, followed by a separate regression for the $\alpha_{i}$, two aggregate bias and trace MSE values are recorded. Examining the individual $\beta_{i}$ and $\gamma_{i j}$, we note that on balance the bias of the $\beta_{i}$ is, in percentage terms, less than that of the $\gamma_{i j}$; compare, for example, the values of $\beta_{1}$ and $\gamma_{11}$ at $T=120$ in both SUR and 3SLS. Note also that the bias does not shrink as $T$ increases, as it would for a consistent estimator. Relative to the $\beta$ and $\gamma$ values, the $\alpha_{i}$ are very poorly estimated, all coefficients being off by a factor of at least two.

The aggregate implications of these patterns are found in the summary statistics $A B$ and $T M$. The 3SLS estimates show uniformly smaller aggregate biases than do the SUR estimates, whereas the trace MSE is uniformly very much larger in 3SLS. There is no obvious reason for the biases of the 3SLS estimates to be less than SUR, but one might conjecture that the IV ef- 
Table 3. Aggregate Bias and Trace Mean Square Error of Price Elasticities: SUR Estimates, Independent Goods Case

\begin{tabular}{|c|c|c|c|c|c|c|c|}
\hline \multirow[b]{2}{*}{ Elasticity } & \multicolumn{3}{|c|}{ No collinearity } & \multicolumn{3}{|c|}{ High collinearity } & \multirow{2}{*}{$\begin{array}{l}\text { Row sum } \\
\& \text { rank }\end{array}$} \\
\hline & $T=30$ & $T=60$ & $T=120$ & $T=30$ & $T=60$ & $T=120$ & \\
\hline \multicolumn{8}{|l|}{ A. Good fit } \\
\hline $\begin{array}{l}A B(A) \\
A B\left(A_{0}\right)\end{array}$ & $\begin{array}{l}0.54564 \\
0.54969\end{array}$ & $\begin{array}{l}0.09908 \\
0.10089\end{array}$ & $\begin{array}{l}0.16321 \\
0.16386\end{array}$ & $\begin{array}{l}0.03885 \\
0.03983\end{array}$ & $\begin{array}{l}0.13688 \\
0.13555\end{array}$ & $\begin{array}{l}0.00987 \\
0.01151\end{array}$ & $\begin{array}{l}0.99353(1) \\
1.00133(3)\end{array}$ \\
\hline$A B\left(L_{3}\right)$ & 0.54986 & 0.10086 & 0.16371 & 0.03955 & 0.13699 & 0.00995 & $1.00092(2)$ \\
\hline$A B\left(L_{2}\right)$ & 0.59753 & 0.10500 & 0.16660 & 0.03965 & 0.13787 & 0.01065 & $1.05729(4)$ \\
\hline$A B\left(L_{1}\right)$ & 0.59891 & 0.10492 & 0.16659 & 0.03968 & 0.13790 & 0.01062 & $1.05862(5)$ \\
\hline$T M(A)$ & 2.48969 & 0.89060 & 0.54941 & 0.08725 & 0.15060 & 0.01480 & $4.18235(1)$ \\
\hline$T M\left(A_{0}\right)$ & 2.50106 & 0.88972 & 0.54983 & 0.08814 & 0.14936 & 0.01638 & $4.19449(3)$ \\
\hline$T M\left(L_{3}\right)$ & 2.50099 & 0.88935 & 0.54986 & 0.08756 & 0.15059 & 0.01490 & $4.19325(2)$ \\
\hline$T M\left(L_{2}\right)$ & 2.58734 & 0.88831 & 0.55224 & 0.08915 & 0.15141 & 0.01529 & $4.28378(4)$ \\
\hline$T M\left(L_{1}\right)$ & 0.59765 & 0.88877 & 0.55239 & 0.08920 & 0.15143 & 0.01528 & $4.29472(5)$ \\
\hline \multicolumn{8}{|l|}{ B. Poor fit } \\
\hline$A B\left(A_{0}\right)$ & 0.24751 & 0.09795 & 0.08681 & 6.82496 & 7.11587 & 2.58226 & $16.95536(1)$ \\
\hline$A B\left(L_{3}\right)$ & 0.24673 & 0.09773 & 0.08600 & 6.83360 & 7.13026 & 2.58580 & $16.98012(3)$ \\
\hline$A B\left(L_{2}\right)$ & 0.26801 & 0.09905 & 0.08768 & 9.05039 & 9.26188 & 2.90218 & $21.66919(4)$ \\
\hline$A B\left(L_{1}\right)$ & 0.26783 & 0.09911 & 0.08771 & 9.48130 & 9.62114 & 2.92181 & $22.47890(5)$ \\
\hline$T M(A)$ & 4.85474 & 2.10746 & 0.97082 & 9.26588 & 8.38408 & 3.29226 & $28.87524(2)$ \\
\hline$T M\left(A_{0}\right)$ & 4.86917 & 2.10094 & 0.96894 & 9.27703 & 8.38041 & 3.28774 & $28.88423(3)$ \\
\hline$T M\left(L_{3}\right)$ & 4.86900 & 0.09663 & 0.96809 & 9.21632 & 8.37602 & 3.28705 & $28.81311(1)$ \\
\hline$T M\left(L_{2}\right)$ & 4.85620 & 2.09437 & 0.96849 & 12.41245 & 11.04842 & 3.71469 & $35.09462(4)$ \\
\hline$T M\left(L_{1}\right)$ & 4.86271 & 2.09506 & 0.96875 & 13.09360 & 11.53978 & 3.74318 & $36.30308(5)$ \\
\hline
\end{tabular}

fect for 3SLS might reduce the correlation between regressors and disturbances even though the instruments are not fully orthogonal to the disturbances. That the trace MSE error is everywhere larger for 3SLS is a direct consequence of the fact that, by construction, the 3SLS variances will be larger than the SUR variances. What is, perhaps, surprising is the factor of ten in the order of magnitude of the differences in SUR and 3SLS trace MSE for the $\beta$ and $\gamma$ coefficients. This suggests that the modest advantage in bias of the 3SLS estimator is more than offset by its larger variance, so that on balance one would not be inclined to estimate the LAIDS model by 3 SLS.

The means and standard deviations of the income and uncompensated own-price elasticities that are generated by these parameter estimates are available in Buse (1993) and I record only the salient points here. The elasticities are nonlinear functions of the parameters and inconsistency of the parameter estimators would in general carry over to the nonlinear functions. The means of the sampling distributions of the ownprice elasticities are surprisingly close to the true values, the proportionate errors of the elasticities typically being much smaller than those of the underlying parameters. The $\eta_{i j}(A)$ estimator gives very satisfactory results despite its dependence on the $\alpha_{i}$, which are, on average, off the mark by a factor of two. Since the $\beta_{i}$ are, on balance, estimated with very small inconsistency, the large upward bias in the $\alpha_{i}$ is apparently offset by an underestimate of the linear combination $\Sigma \gamma_{i j} \ln p_{k}$; see (15). Finally, as in table 2, the 3SLS estimates produce smaller biases but any gains relative to SUR are offset by the very large sampling variances. The regularities we have noted apply almost without exception to the remaining experiments and these will therefore be discussed using aggregate bias and trace MSE only. Alston, Foster, and Green have conducted a Monte Carlo investigation on price elasticity formulas using both SUR and 3SLS. They found no substantive differences between the two estimators. Later I will offer some conjectures as to why my conclusions differ from theirs. The biases of the income elasticities for some goods were found to be substantial at $T=30$ and 60 but were quite small at $T=120$ for all goods. The differences in the means of the three elasticities $\eta_{x}(A), \eta_{x}\left(L_{1}\right)$ and $\eta_{x}\left(L_{2}\right)$ were, however, very small.

Tables 3 and 4 record the aggregate bias and 
Table 4. Aggregate Bias and Trace Mean Square Error of Price Elasticities: SUR Estimates, Complementary Goods Case

\begin{tabular}{|c|c|c|c|c|c|c|c|}
\hline \multirow[b]{2}{*}{ Elasticity } & \multicolumn{3}{|c|}{ No collinearity } & \multicolumn{3}{|c|}{ High collinearity } & \multirow{2}{*}{$\begin{array}{l}\text { Row sum } \\
\& \text { rank }\end{array}$} \\
\hline & $T=30$ & $T=60$ & $T=120$ & $T=30$ & $T=60$ & $T=120$ & \\
\hline \multicolumn{8}{|l|}{ A. Good fit } \\
\hline$A B\left(A_{0}\right)$ & 0.17308 & 0.15994 & 0.36676 & 0.03992 & 0.21832 & 0.24867 & $1.20669(2)$ \\
\hline$A B\left(L_{3}\right)$ & 0.17719 & 0.16142 & 0.36663 & 0.03785 & 0.21700 & 0.24826 & $1.20835(3)$ \\
\hline$A B\left(L_{2}\right)$ & 0.18568 & 0.16368 & 0.36761 & 0.03654 & 0.21657 & 0.24971 & $1.21979(5)$ \\
\hline$A B\left(L_{1}\right)$ & 0.18503 & 0.16361 & 0.36763 & 0.03643 & 0.21655 & 0.24970 & $1.21895(4)$ \\
\hline$T M(A)$ & 0.25335 & 0.20484 & 0.37799 & 0.11293 & 0.23668 & 0.26194 & $1.44773(1)$ \\
\hline$T M\left(A_{0}\right)$ & 0.25448 & 0.20508 & 0.37846 & 0.11398 & 0.23780 & 0.26218 & $1.45198(3)$ \\
\hline$T M\left(L_{3}\right)$ & 0.25523 & 0.20533 & 0.37804 & 0.11276 & 0.23671 & 0.26186 & $1.44993(2)$ \\
\hline$T M\left(L_{2}\right)$ & 0.26025 & 0.20728 & 0.37945 & 0.11825 & 0.23666 & 0.26302 & $1.46491(4)$ \\
\hline$T M\left(L_{1}\right)$ & 0.26035 & 0.20727 & 0.37947 & 0.11874 & 0.23666 & 0.26300 & $1.46449(5)$ \\
\hline \multicolumn{8}{|l|}{ B. Poor fit } \\
\hline$A B(A)$ & 13.15361 & 6.59695 & 4.50433 & 2.40880 & 0.58750 & 0.67183 & $7.92302(2)$ \\
\hline$A B\left(A_{0}\right)$ & 13.30965 & 6.69743 & 4.58662 & 2.37995 & 0.58142 & 0.67056 & $28.22563(3)$ \\
\hline$A B\left(L_{3}\right)$ & 12.97557 & 6.56320 & 4.50265 & 2.40771 & 0.58772 & 0.67177 & $27.70862(1)$ \\
\hline$A B\left(L_{2}\right)$ & 25.59179 & 8.73540 & 5.72244 & 4.05274 & 0.76992 & 0.67947 & $45.55176(4)$ \\
\hline$A B\left(L_{1}\right)$ & 40.28107 & 9.28235 & 5.98347 & 4.74433 & 0.79895 & 0.67960 & $61.76977(5)$ \\
\hline$T M(A)$ & 13.61214 & 7.01682 & 4.69599 & 2.80234 & 0.84355 & 0.89313 & $29.86397(2)$ \\
\hline$T M\left(A_{0}\right)$ & 13.75442 & 7.12524 & 4.78316 & 2.76947 & 0.83478 & 0.89051 & $30.15758(3)$ \\
\hline$T M\left(L_{3}\right)$ & 13.38554 & 6.97827 & 4.69306 & 2.79467 & 0.84302 & 0.89307 & $29.59063(3)$ \\
\hline$T M\left(L_{2}\right)$ & 26.93418 & 9.44476 & 6.03723 & 4.80458 & 1.11881 & 0.90463 & $49.24419(4)$ \\
\hline$T M\left(L_{1}\right)$ & 44.34529 & 10.18070 & 6.34069 & 5.73672 & 1.16761 & 0.90491 & $68.60392(5)$ \\
\hline
\end{tabular}

trace MSE of SUR estimates of the price elasticities in the independent and complementary goods cases, respectively. ${ }^{8}$ In table 3 , the evidence on the entire vector of uncompensated own- and cross-price elasticities is not decisively in favor of the conventional AIDS formula. On aggregate bias, $\eta(A)$ ranks first seven of twelve times and on trace MSE it ranks five of twelve times. It also ranks last a number of times according to both criteria. The GreenAlston elasticity $\eta\left(L_{1}\right)$ ranks third once, and fourth and fifth the rest of the time on aggregate bias. On trace MSE, $\eta\left(L_{1}\right)$ ranks second twice and last seven times. There are indications that both $\eta\left(A_{0}\right)$ and $\eta\left(L_{3}\right)$ do well in certain cases and this shows up in the row sums of the "poor" fit specification, in which both elasticities capture first-place ranking once. When the row sums are added across the two fit specifications we obtain the following rankings on total aggregate bias and trace MSE:

$$
A B \text { (1) } \eta\left(A_{0}\right)=17.9567
$$

\footnotetext{
${ }^{8}$ The performance of the income elasticities was also evaluated. The rankings, on both aggregate bias and trace MSE, were (1) $\eta_{x}(A) ;(2) \eta_{x}\left(L_{2}\right)$; (3) $\eta_{x}\left(L_{1}\right)$. Details are given in Buse (1993).
}
(2) $\eta(A)=17.9590$
(3) $\eta\left(L_{3}\right)=17.9810$
(4) $\eta\left(L_{2}\right)=22.7265$
(5) $\eta\left(L_{1}\right)=23.5375$
$T M(1) \eta\left(L_{3}\right)=33.0064$
(2) $\eta(A)=33.0576$
(3) $\eta\left(A_{0}\right)=33.0787$
(4) $\eta\left(L_{2}\right)=39.3784$
(5) $\eta\left(L_{1}\right)=40.5978$.

The differences between the three leading elasticities are of an order of magnitude that suggest that any one of them might serve as an appropriate elasticity calculation. Insofar as $\eta\left(A_{0}\right)$ and $\eta\left(L_{3}\right)$ do not require estimation of the $\alpha$ parameters, they have the advantage of computational simplicity.

If we aggregate the row sums for the price elasticity formulas in table 4 , we obtain the following rankings:
$A B(1) \eta\left(L_{3}\right)=28.9176$
(2) $\eta(A)=29.1268$
(3) $\eta\left(A_{0}\right)=29.4323$
(4) $\eta\left(L_{2}\right)=46.7716$
(5) $\eta\left(L_{1}\right)=62.9887$
$T M(1) \eta\left(L_{3}\right)=31.0406$
(2) $\eta(A)=31.3117$
(3) $\eta\left(A_{0}\right)=31.6096$
(4) $\eta\left(L_{2}\right)=50.7091$
(5) $\eta\left(L_{1}\right)=70.0694$.

Apart from the transposition of $\eta\left(A_{0}\right)$ and $\eta\left(L_{3}\right)$ in the first and third position under $A B$, these 
rankings are identical to those observed in table 3 . Furthermore, as in table 3 , the aggregate statistics show that the elasticity formulas fall into two groups, with the two LAIDS elasticities, $\eta\left(L_{1}\right)$ and $\eta\left(L_{2}\right)$, being the unambiguously inferior group.

These rankings do not correspond to those obtained by Foster, Green, and Alston in their Monte Carlo study. They ranked $\eta\left(L_{1}\right)$ marginally ahead of $\eta\left(L_{3}\right)$, whereas my results lead to the unambiguous conclusion that $\eta\left(L_{1}\right)$ is inferior. Their Monte Carlo design differed from mine in that their prices were normal and not autocorrelated, the utility level was held constant, and the negativity restriction was not used. Any one of these conditions could account for the differences in results, but I am inclined to believe that the principal reason for the divergence in rankings is the manner in which Foster, Green, and Alston calculated $\mathrm{E}\left(w_{i}\right)$. They used the actual sample median prices to determine $\mathrm{E}\left(w_{i}\right)$ rather than using appropriate population values. I believe my procedure is the correct one.

To conclude my discussion of tables 3 and 4, two broad patterns should be noted. First, the trace MSE increases with increased disturbance variance, as it should, but there is also some tendency for the bias to increase as well. This is consistent with my analytical discussion, where I established that variance of the log measurement error, $\xi_{t}$, increases with increased variances of the disturbances [see (10)]. The larger variance in the measurement error will induce larger inconsistency in the estimator, which shows up in the bias statistic. The second regularity is the tendency of both bias and MSE to decline as we move to high collinearity in the data; the exception is the poor fit specification in table 4. This regularity appears to support the Deaton and Muellbauer conjecture that high price collinearity will lead to a good approximation of $\ln P_{t}$ by $\ln P_{t}^{*}$ and hence good estimates. Curiously, this regularity is not dependent on a high $\mathrm{R}^{2}$ between $\ln P_{t}$ and $\ln P_{t}^{*}$. In table 3 , the average correlation between $P_{t}$ and $P_{t}^{*}$ is greater than 0.99 for all experiments, whereas in table 4 the highest correlation is 0.7294 . Yet in table 4 , there is a well-defined improvement in the $A B$ and $T M$ measures as one moves from zero to high collinearity. It would appear that the role of collinearity is important, but the mechanism which generates this role remains obscure. We should note that the role of the substitution matrix [and hence via (29) the $\gamma$ parameter] appears to be more important than the disturbance variances in determining the correlation between $P_{t}$ and $P_{t}^{*}$. There are only very small decreases in this correlation as the variance goes from low to high, but there is a very large decrease in the correlation as we move from the independent good to the complementary goods specification of the substitution matrix.

\section{Conclusions}

I set out to explore two issues: the properties of some LAIDS estimators and the associated elasticities. Given the pre-eminence of SUR as an estimator of the LAIDS model, it is something of a surprise to find that its small-sample performance has never been investigated in this context. The commonly expressed view that SUR is consistent and asymptotically efficient when iterated to the ML solution has been demonstrated to be false and the inconsistency confirmed by my Monte Carlo study. The results show that this inconsistency is not trivial. Deviations from true parameter values as large as $20 \%$ are quite common at samples as large as 120. Although the 3SLS estimator had smaller biases, its use, because of its large sampling variability, is not recommended. For example, if the standard deviations of the sampling distribution of 3SLS were used to test the null $\beta_{\mathrm{i}}=$ 0 (homotheticity), that null would not be rejected even though it is false, a sample size of 120 notwithstanding. This mistake would not occur if the SUR estimates were used in the test. In light of the inconsistency of both SUR and 3SLS, we must ask whether the LAIDS model should be estimated at all. It is clear that the computational simplicity of linearity can exact a substantial cost. Given the speed and power of the nonlinear algorithms available today, full ML estimation of the complete AIDS model may be a feasible alternative, at least for modest-sized systems. I plan to explore the properties of both the ML estimator and the iterated Browning-Meghir estimator in another paper.

The results on elasticities are, up to a point, unambiguous. Income elasticities constructed specifically for the LAIDS model are inferior to the conventional formula derived from the nonlinear model. Neither the Green-Alston elasticities nor the alternative, $\eta_{i j}\left(L_{2}\right)$, developed in the present paper can be recommended. Papers such as Lin and Makus; and Carriker, Featherstone, and Schroeder, which have used 
the Green-Alston elasticities in the belief that they represent an improvement over the conventional methods, are likely to make faulty inferences about the sizes of price elasticities. Of the other methods that I have examined, it is not possible to make a decisive discrimination, the summary statistics being practically identical. Insofar as the most widely used method, $\eta_{i j}\left(L_{3}\right)$, is marginally the best, there is no compelling reason to change current practice. On the other hand, the results do show conclusively that the conventional AIDS elasticity and the modification $\eta_{i j}\left(A_{0}\right)$ proposed in this paper are quite appropriate for use in the LAIDS model.

[Received May 1992; final revision received March 1994.]

\section{References}

Alston, J.M., K.A. Foster, and R.D. Green. "Estimating Elasticities with the Linear Approximate Almost Ideal Demand System: Some Monte Carlo Results." Rev. Econ. and Statist. 76(May 1994):351-56.

Aitchison, J., and J.A.C. Brown. The Lognormal Distribution. Cambridge: Cambridge University Press, 1957.

Blanciforti, L.A., R.D. Green, and G.A. King. "U.S Consumer Behavior Over the Postware Period: An Almost Ideal Demand System Analysis." University of California Giannini Foundation Monograph No. 40, 1986.

Browning, M., and C. Meghir. "The Effects of Male and Female Labor Supply on Commodity Demands." Econometrica 59(July 1991):925-52.

Buse, A. "Consistent and Efficient Estimation of the Almost Ideal Demand System." Paper presented to Canadian Econometrics Study Group, 25-27 September 1992. "Evaluating the Linearized Almost Ideal De- mand System." University of Alberta Research Paper 93-18, August 1993.

Carriker, G.C., A.M. Featherstone, and T.C. Schroeder. "Farm Household Consumption Patterns." Rev. Agr. Econ. 15(January 1993):63-73.

Chalfant, J.A. "A Globally Flexible, Almost Ideal Demand System.” J. Bus. Econ. Statist. 5(April 1987):233-42.

Deaton, A., and J. Muellbauer. "An Almost Ideal Demand System." Amer. Econ. Rev. 70(June 1980):312-26.

Eales, J.S., and L. Unnevehr. "Structural Change in U.S. Meat Demand." Amer. J. Agr. Econ. 75(May 1993):259-68.

Fulponi, L. "The Almost Ideal Demand System: An Application to Food and Meat Groups for France." J. Agr. Econ. 40(March 1983):82-92.

Goddard, D. "An Analysis of Canadian Aggregate Demand for Food at Home and Away from Home." Can. J. Agr. Econ. 31(November 1983):289-318.

Green, R., and J.M. Alston. "Elasticities in AIDS Models." Amer. J. Agr. Econ. 72(May 1990):442-45.

. "Elasticities in AIDS Models: A Clarification and Extension." Amer. J. Agr. Econ. 73(May 1991):874-75.

Haden, K. "The Demand for Cigarettes in Japan." Amer. J. Agr. Econ. 72(May 1990):446-50.

Lin, B.H., and L.D. Makus. "Japanese Barley Imports: Implications of the 1988 Japanese Beef Market Access Agreement." Can. J. Agr. Econ. 39(November 1991):423-37.

Mergos, G.J., and G.S. Donatos. "Consumer Behavior in Greece: An Application of the Almost Ideal Demand System." App. Econ. 21(July 1989):973-93.

Murfin, A. "Market Share in the UK Passenger Car Market: Marketing, Expenditure and Price Effects." App. Econ. (August 1984):611-32.

Pashardes, P. "Bias in Estimating the Almost Ideal Demand System with the Stone Index Approximation.” Econ. J. 103(June 1993):908-15. 